\title{
Glucagon-Like Peptide-1 Infusion Suppresses Aldosterone Levels in Healthy Normal-Weight Individuals: Double-Blind, Placebo-Controlled Crossover Study
}

\author{
Maja Baretić (D) · Vesna Kušec · Ivana Pavlić-Renar
}

Received: July 17, 2018 / Published online: October 8, 2018

(C) The Author(s) 2018
Enhanced Digital Features To view enhanced digital features for this article go to https://doi.org/10.6084/ m9.figshare.7111430.

M. Baretić $(\bowtie) \cdot$ I. Pavlić-Renar

Department of Endocrinology, University Hospital

Centre Zagreb, Zagreb, Croatia

e-mail: Maja.baretic@kbc-zagreb.hr;

Maja.simek@zg.t-com.hr

V. Kušec

Clinical Institute of Laboratory Diagnosis,

University Hospital Centre Zagreb, Zagreb, Croatia

M. Baretić · V. Kušec · I. Pavlić-Renar

School of Medicine, University of Zagreb, Zagreb, Croatia native gut hormone GLP-1 influences the renin-angiotensin-aldosterone system (RAAS).

Methods: Fourteen healthy participants (6 males and 8 females) were included in a doubleblind, placebo-controlled crossover study. After overnight fasting and oral sodium loading, participants were randomly assigned to receive either placebo (500 $\mathrm{ml}$ of $0.9 \%$ saline) or GLP-1 infusion $(1.5 \mathrm{pmol} / \mathrm{kg} / \mathrm{min}$ dissolved in $500 \mathrm{ml}$ of $0.9 \%$ saline) over a 3 -h period. After 3 and $6 \mathrm{~h}$, the following parameters were measured: glucose, insulin, plasma renin activity, aldosterone, GLP-1, and antidiuretic hormone. After 7 days, the protocol was repeated, except that those who had previously received placebo now received GLP-1 infusion, and those who had previously received GLP-1 now received placebo.

Results: Three hours after GLP-1 infusion, aldosterone had decreased by a statistically significant amount $(p<0.008)$ compared to the baseline level.

Conclusion: The present study showed that native GLP-1 can decrease aldosterone secretion in a group of healthy individuals, supporting the idea of beneficial outcomes of GLP-1-activating agents on blood pressure and the RAAS. Trial Registration: ClinicalTrials.gov Identifier: NCT02130778.

Keywords: Aldosterone; Diabetes; GLP-1; Kidney; Nephropathy 


\section{INTRODUCTION}

Diabetes is the most common cause of end-stage renal disease in the developed world [1]. The pathophysiology of diabetic kidney disease is composite: acquired metabolic and hemodynamic changes associated with genetic traits activate prooxidative, proinflammatory, and profibrotic pathways leading to hyperfiltration. Hyperfiltration is followed by a gradual decline in glomerular filtration [2].

The incretin hormone glucagon-like peptide1 (GLP-1) is released from intestinal enteroendocrine L cells in response to a carbohydrate meal. GLP-1 has many effects on the human body, but its glucose-lowering effect due to its stimulation of insulin secretion is the most important. GLP-1 acts on renal function and hemodynamics, and GLP-1 receptors are widely expressed in many tissues beyond the pancreas, including the kidneys. In humans, receptors have been found in large and medium-sized renal arteries but not in the tubules or glomeruli [3].

Incretin-based therapy for diabetes lowers systolic blood pressure, as also confirmed by a large meta-analysis drawing from six clinical trials and comprising 2171 subjects treated for at least 6 years with a GLP-1 receptor agonistexenatide [4]. The blood pressure-lowering effect was later attributed to improved endothelial dysfunction through the GLP-1 receptor-dependent pathway, stimulating diuresis and natriuresis [5]. Hypoglycemic agents that utilize the effect of incretin also have renoprotective properties independent of their glucose-lowering effects.

In experimental animal models, exenatide suppressed the inflammatory mediator nuclear factor kappa B, reduced substances associated with abnormalities in vascular function, and delayed the progression of diabetic renal disease [6]. Liraglutide, another GLP-1 receptor agonist, was found to be protective against increased renal oxidative stress under chronic hyperglycemia in GLP-1 knockout mice [7].

In experimental studies, prolonged administration of exendin-4 (a peptide agonist of GLP1) in normal rats resulted in increased plasma concentrations of aldosterone and corticosterone, independent of adrenocorticotropic hormone (ACTH). In streptozotocin-induced diabetic rats, exendin- 4 increased the plasma levels of ACTH along with aldosterone and corticosterone. The effect in normal rats was attributed to aldosterone and corticosterone secretion by exendin- 4 but is not mediated through the hypothalamo-hypophyseal axis [8].

The antihypertensive and renoprotective effects of GLP-1 receptor agonists are partly explained by their vasoactive effect and the increased natriuresis that results from their inhibition of the sodium-hydrogen ion exchanger in the proximal tubule. Additional effects on the renin-angiotensin-aldosterone system (RAAS) may also contribute to renal benefits $[9,10]$.

The action of GLP-1 on kidney function is still not entirely clear, and the few studies that have been conducted in humans have confirmed the previously mentioned natriuretic effect and the influence on fluid homeostasis and the RAAS [11-13].

The aim of this study was to investigate the effect of GLP-1 infusion after oral and intravenous sodium loading on kidney hemodynamics and the RAAS, particularly aldosterone, in healthy participants with normal body weight.

\section{METHODS}

\section{Participants}

The study was conducted at the Department of Endocrinology, Internal Clinic, University Hospital Centre Zagreb, and registered at ClinicalTrials.gov with the identifier NCT02130778. All procedures performed in studies involving human participants were carried out in accordance with the ethics committee of University Hospital Centre Zagreb and with the 1964 Helsinki Declaration and its later amendments or comparable ethical standards. Informed consent was obtained from all participants included in the study.

Fourteen healthy non-obese $(\mathrm{BMI}<30)$ participants (6 males and 8 females, mean age 
Table 1 Descriptive statistics for parameters measured at baseline (visit 1)

\begin{tabular}{lrrrrrr}
\hline & Mean & \multicolumn{1}{c}{ SD } & Median & Minimum & Maximum & $\boldsymbol{N}$ \\
\hline Age (years) & 35.50 & 7.62 & 33.00 & 26.00 & 48.00 & 14 \\
Syst BP (mmHg) & 118.57 & 10.71 & 115.00 & 103.00 & 137.00 & 14 \\
Dia BP (mmHg) & 70.29 & 10.37 & 71.00 & 42.00 & 85.00 & 14 \\
Syst dip (mmHg) & 12.43 & 4.26 & 12.50 & 6.00 & 19.00 & 14 \\
Dia dip (mmHg) & 16.71 & 7.07 & 15.50 & 5.00 & 31.00 & 14 \\
Total body water \% & 53.96 & 3.67 & 54.20 & 47.00 & 59.00 & 14 \\
BMI (kg/m $\left.{ }^{2}\right)$ & 23.15 & 2.77 & 22.85 & 18.10 & 28.40 & 14 \\
Fat \% & 22.04 & 6.00 & 19.90 & 14.00 & 32.80 & 14 \\
Fat mass (kg) & 15.30 & 4.61 & 16.45 & 7.90 & 23.00 & 14 \\
Muscle mass (kg) & 51.56 & 11.27 & 46.95 & 38.00 & 77.00 & 14 \\
Visceral fat rating & 3.93 & 2.37 & 4.00 & 1.00 & 8.00 & 14 \\
OGTT GLC1 (mmol/l) & 4.40 & 0.40 & 4.35 & 3.80 & 5.10 & 14 \\
OGTT GLC2 (mmol/l) & 3.90 & 0.62 & 3.90 & 2.70 & 4.80 & 14 \\
GLP-1 visit 1 (pmol/l) & 3.29 & 5.92 & 0.85 & 0.00 & 17.00 & 14 \\
TSH visit 1 (mIU/l) & 1.41 & 0.66 & 1.37 & 0.50 & 2.90 & 14 \\
\hline
\end{tabular}

$S D$ standard deviation, BP blood pressure, Syst systolic, Dia diastolic, Dip dipper, BMI body mass index, OGTT oral glucose tolerance test, GLC glucose, GLP-1 glucagon-like peptide-1, TSH thyrotropin hormone

33 years, range 29-64 years) were randomized for a double-blind, placebo-controlled, crossover study. The participants within the group of healthy volunteers were randomized. Sample size was not calculated prior to the start of the study. The sample size was in line with those used in previous studies performed with a similar protocol which included 12-16 participants [11-13].

Table 1 shows the descriptive statistics of parameters for the study participants measured at baseline (visit 1).

Inclusion criteria were: age $18-50$ years, body mass index (BMI) $18-27 \mathrm{~kg} / \mathrm{m}^{2}$ (for those with BMI $>25 \mathrm{~kg} / \mathrm{m}^{2}$, fat percentage was normal for gender and age), and, for women, a low likelihood of conception prior to or during the study. Participants were healthy, as determined by fasting blood parameters of the total blood count, standard biochemistry tests, thyroidstimulating hormone, urine chemistry, oral glucose tolerance test (OGTT), 24-h urine protein excretion, and glomerular filtration assessment. Standard biochemical methods were usedto measure laboratory parameters. On the first visit, the following baseline tests were administered: fasting glucose and insulin, GLP1 , aldosterone, PRA (plasma renin activity), and antidiuretic hormone (ADH).

All participants had normal findings on standard 12-lead electrocardiogram, kidney ultrasound, and 24-h blood pressure monitoring. None of the participants had any history of hypertension, diabetes, kidney disease, myocardial infarction, cerebrovascular incidents, transient ischemic attack, or malignancy within the last 5 years; they also were not currently in a febrile state or pregnant/nursing.

Body composition and hydration were estimated by means of bioelectrical impedance analysis [total body water (TBW), fat percentage, fat mass, muscle mass, and visceral fat 
rating (VFR)]. Bioimpedance was measured using a Tanita body composition analyzer (model SC-330, Tanita Europe BV, Amsterdam, Netherlands).

\section{Study Design}

Before being enrolled in the study (screening), participants completed a medical history interview. They were instructed to come the following day for their first visit while fasting.

Randomization was performed by a coworker not included in the study (8 patients were randomized to receive infusion with GLP-1 first and than placebo; another 7 patients were randomized to receive placebo first and then infusion with GLP-1).

The study protocol included two visits separated by at least 7 days.

Blood was drawn for laboratory analysis and routine physical examination.

On the second visit day, participants arrived at the research unit in the morning after an overnight fast. Initially, an oral saline load was delivered in the form of a standardized meal (soup). The soup was consumed in $30 \mathrm{~min}$. After that, the participants were instructed to drink $1 / 21$ of bottled water during the following $1 \mathrm{~h}$. In addition to the soup and water, a saline infusion with or without GLP-1 $(1.5 \mathrm{pmol} / \mathrm{kg} /$ min) was given at a rate of $0.06 \mathrm{ml} / \mathrm{kg} / \mathrm{min}$ for $3 \mathrm{~h}$ from the beginning of the oral sodium load. The investigators and nurses performing the procedure were blinded to the test conditions; the solutions were prepared by a technician not involved with the study participants. Blood samples were drawn 180 and $360 \mathrm{~min}$ from the beginning of infusion (Fig. 1). The following parameters were measured at both time points: glucose, insulin, PRA, aldosterone, GLP-1, and $\mathrm{ADH}$. After $6 \mathrm{~h}$, the sodium, chloride, calcium, creatinine, and osmolality were measured in urine.

Patients were instructed to return after at least 7 days for a third visit, fasting as before. The same procedure carried out in visit 2 was performed at visit 3, but if they received a saline infusion with GLP-1 at visit 2 they received a saline infusion without GLP-1 at visit 3, and vice versa.

\section{Materials}

Synthetic human GLP-1 was obtained from Bachem (Bubendorf, Switzerland). Peptide content was administered at a calculated dosage of $1.5 \mathrm{pmol} / \mathrm{kg} / \mathrm{min}$ dissolved in $500 \mathrm{ml} 0.9 \%$ saline solution. The placebo infusion was $0.9 \%$ saline solution. The oral sodium load was delivered in the form of 0.51 soup containing $12 \mathrm{~g} \mathrm{NaCl}$ (it contained $4680 \mathrm{mg}$, i.e., $203,58 \mathrm{mmol} / \mathrm{l}$ of sodium), which is approximately the average daily intake of salt per adult in Croatia [14]. The ingested soup included virtually no fat, proteins, or sugar, and only a small amount of flavor enhancer (monosodium glutamate) and artificial food coloring. The soup concentrate was dissolved in standard bottled water. Bottled water of the same brand $(500 \mathrm{ml}$ of natural artesian water, calcium/magnesium ratio 2:1, pH 7.4) was served as a beverage after the soup.

Blood for GLP-1 assessment was drawn through an antecubital cannula into syringes with EDTA (6 mol/l) and aprotinin (1000 kIU/l) and kept on ice. After centrifugation, the plasma samples were kept frozen at $-20^{\circ} \mathrm{C}$ until analysis. GLP-1 was determined using the Active GLP-1 (7-36) ELISA ALPCO (Salem, USA) diagnostic kit.

Blood for ADH assessment was drawn through an antecubital cannula into syringes and kept on ice. After centrifugation, the plasma samples were kept frozen at $-20^{\circ} \mathrm{C}$ until analysis. ADH was determined using Cayman Chemical's (Ann Arbor, Michigan, USA) arginine vasopressin EIA diagnostic kit.

\section{Methods}

The data were analyzed using descriptive statistics, the chi-square test, the two-sample $t$ test, analysis of variance (ANOVA), and correlation analysis. Descriptive statistics were used to describe the basic features of the sample in the study (proportions for categorical data and mean \pm standard deviation for normally 


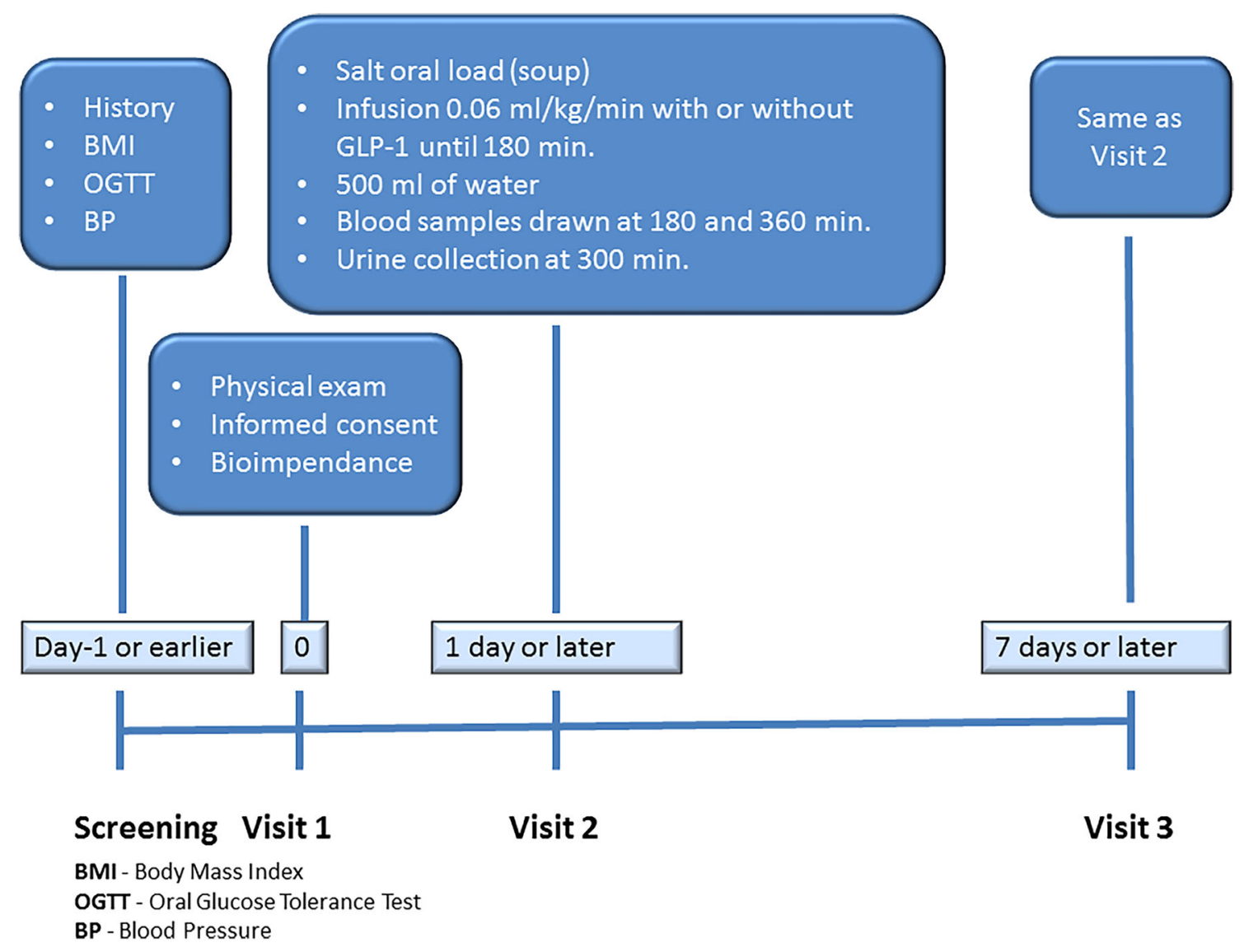

Fig. 1 Study protocol

distributed continuous variables, or median and interquartile range for variables deviating from the normal distribution). For continuous variables, the independent $t$-test or ANOVA (for two or more groups) was used. For repeated measures (paired samples), we used the paired $t$-test for two measurements or a repeated-measures ANOVA for three or more measurements. Statistical analysis was performed using SPSS 15.0 (SPSS, Chicago, USA), with the level of statistical significance set at $p<0.05$.

\section{RESULTS}

Some of the participants had baseline (at visit 1) $\mathrm{ADH}$ and aldosterone levels above the normal ranges. Mean aldosterone was $241.57 \mathrm{pmol} / \mathrm{l}$ (normal range 96-541), and two participants had basal aldosterone above the upper limit, though the presence of normal PRA/aldosterone excluded primary hyperaldosteronism in these individuals. Mean ADH was $2.08 \mathrm{pg} / \mathrm{ml}$ (normal range 0.4-5.2), and one participant had an $\mathrm{ADH}$ level above the normal range, but normal serum baseline electrolyte levels excluded inappropriate $\mathrm{ADH}$ secretion syndrome in this participant.

Biochemical parameters at baseline (visit 1) and at 3 and $6 \mathrm{~h}$ in patients receiving the placebo, and in patients receiving the GLP-1 infusion, were compared by repeated-measures ANOVA (Table 2). After GLP-1 infusion, aldosterone decreased by a statistically significant amount $(p<0.008)$ after $3 \mathrm{~h}$ compared to the baseline level (Fig. 2).

Insulin also decreased by a statistically significant amount $3 \mathrm{~h}$ after GLP-1 infusion $(p<0.001)$ and $6 \mathrm{~h}$ after saline infusion $(p<0.001)$ compared to the baseline level.

No statistically significant differences were observed in any of the other measured parameters considered in the study. 
Table 2 Results of repeated-measures ANOVA of the measured parameters comparing values obtained at baseline (visit 1), 3 , and $6 \mathrm{~h}$ after saline infusion and after GLP-1 infusion

\begin{tabular}{|c|c|c|c|c|c|c|c|}
\hline & Mean & SD & Median & Min & Max & $N$ & $F$ ratio, $p$ value \\
\hline Aldo visit 1 (pmol/l) & 241.57 & 113.43 & $219.00^{*}$ & 96.00 & 541.00 & 14 & $F(2.5 .29 .3)=5.414$. \\
\hline Aldo saline $3 \mathrm{~h}(\mathrm{pmol} / \mathrm{l})$ & 134.64 & 52.82 & 133.50 & 82.00 & 256.00 & 14 & $p=0.008$ \\
\hline Aldo saline $6 \mathrm{~h}(\mathrm{pmol} / \mathrm{l})$ & 154.64 & 65.40 & 140.00 & 80.00 & 291.00 & 14 & \\
\hline Aldo GLP-1 3 h (pmol/l) & 136.71 & 50.38 & $129.00^{*}$ & 82.00 & 234.00 & 14 & \\
\hline Aldo GLP-1 6 h (pmol/l) & 182.93 & 107.99 & 150.50 & 86.00 & 466.00 & 14 & \\
\hline PRA visit $1(\mathrm{ug} / \mathrm{l} / \mathrm{h})$ & 0.62 & 0.30 & 0.55 & 0.20 & 1.30 & 14 & $F(4.52)=2.078$ \\
\hline PRA saline $3 \mathrm{~h}$ (ug/l/h) & 0.72 & 0.41 & 0.65 & 0.20 & 1.50 & 14 & $p=0.097$ \\
\hline PRA saline $6 \mathrm{~h}(\mathrm{ug} / \mathrm{l} / \mathrm{h})$ & 0.87 & 0.49 & 0.80 & 0.30 & 1.70 & 14 & \\
\hline PRA GLP-1 3 h (ug/l/h) & 0.51 & 0.26 & 0.50 & 0.10 & 1.20 & 14 & \\
\hline PRA GLP-1 6 h (ug/l/h) & 0.72 & 0.47 & 0.71 & 0.10 & 2.00 & 14 & \\
\hline $\mathrm{ADH}$ visit $1(\mathrm{pg} / \mathrm{ml})$ & 2.08 & 338.22 & 122.00 & 45.00 & 1371.00 & 14 & $F(1.9 .25 .2)=2.410$ \\
\hline $\mathrm{ADH}$ saline $3 \mathrm{~h}(\mathrm{pg} / \mathrm{ml})$ & 1.24 & 66.95 & 108.00 & 44.00 & 254.00 & 14 & $p=0.112$ \\
\hline ADH saline $6 \mathrm{~h}(\mathrm{pg} / \mathrm{ml})$ & 2.23 & 389.95 & 88.50 & 41.00 & 1554.00 & 14 & \\
\hline ADH GLP-1 3 h (pg/ml) & 5.24 & 794.99 & 122.00 & 43.00 & 2304.00 & 14 & \\
\hline ADH GLP-1 6 h (pg/ml) & 3.48 & 434.61 & 125.00 & 41.00 & 1267.00 & 14 & \\
\hline Insulin visit $1(\mathrm{mU} / \mathrm{l})$ & 7.48 & 3.49 & $6.90^{*}$ & 3.00 & 17.00 & 14 & $F(1.8 .23 .9)=9.225$ \\
\hline Insulin saline $3 \mathrm{~h}(\mathrm{mU} / \mathrm{l})$ & 4.2 & 1.75 & 4.2 & 1.3 & 7.7 & 14 & $p=0.001$ \\
\hline Insulin saline $6 \mathrm{~h}(\mathrm{mU} / \mathrm{l})$ & 4.16 & 1.5 & $4.10^{*}$ & 2.3 & 7.6 & 14 & \\
\hline Insulin GLP-1 $3 \mathrm{~h}(\mathrm{mU} / \mathrm{l})$ & 3.89 & 1.49 & 4.03 & 1.4 & 7.1 & 14 & \\
\hline Insulin GLP-1 $6 \mathrm{~h}(\mathrm{mU} / \mathrm{l})$ & 4.64 & 2.23 & $4.00^{*}$ & 2.2 & 9.5 & 14 & \\
\hline Na GLP-1 & 132.36 & 73.28 & 119.00 & 29.00 & 245.00 & 14 & $F(1.13)=1.832$ \\
\hline $\mathrm{Na}$ in saline & 112.14 & 73.92 & 93.00 & 34.00 & 250.00 & 14 & $p=0.199$ \\
\hline
\end{tabular}

Bold measures are showing values with statistical significance

Aldo aldosterone, PRA plasma renin activity, $A D H$ antidiuretic hormone, GLP-1 glucagon-like peptide-1, $S D$ standard deviation

There was no positive correlation between the observed change in aldosterone level (aldosterone level $3 \mathrm{~h}$ after GLP-1 infusion compared to baseline) and any of the following variables: age, average systolic blood pressure, average diastolic blood pressure, overnight systolic dipper and overnight diastolic dipper percentages, TSH, glucose in OGTT (glucose at first or second time point), base value of GLP-1, BMI, mean fat percentage, fat mass, muscle mass, VFR, or TBW.
No statistically significant correlations or sex-related differences were found between any of the tested parameters.

There was a significant decrease in median insulin during placebo infusion after $6 \mathrm{~h}$ $(p=0.011)$ and a decrease in median insulin during GLP-1 infusion after $3 \mathrm{~h}(p=0.016)$ compared to the baseline insulin level. There was no positive correlation between the observed change in insulin level (insulin level 


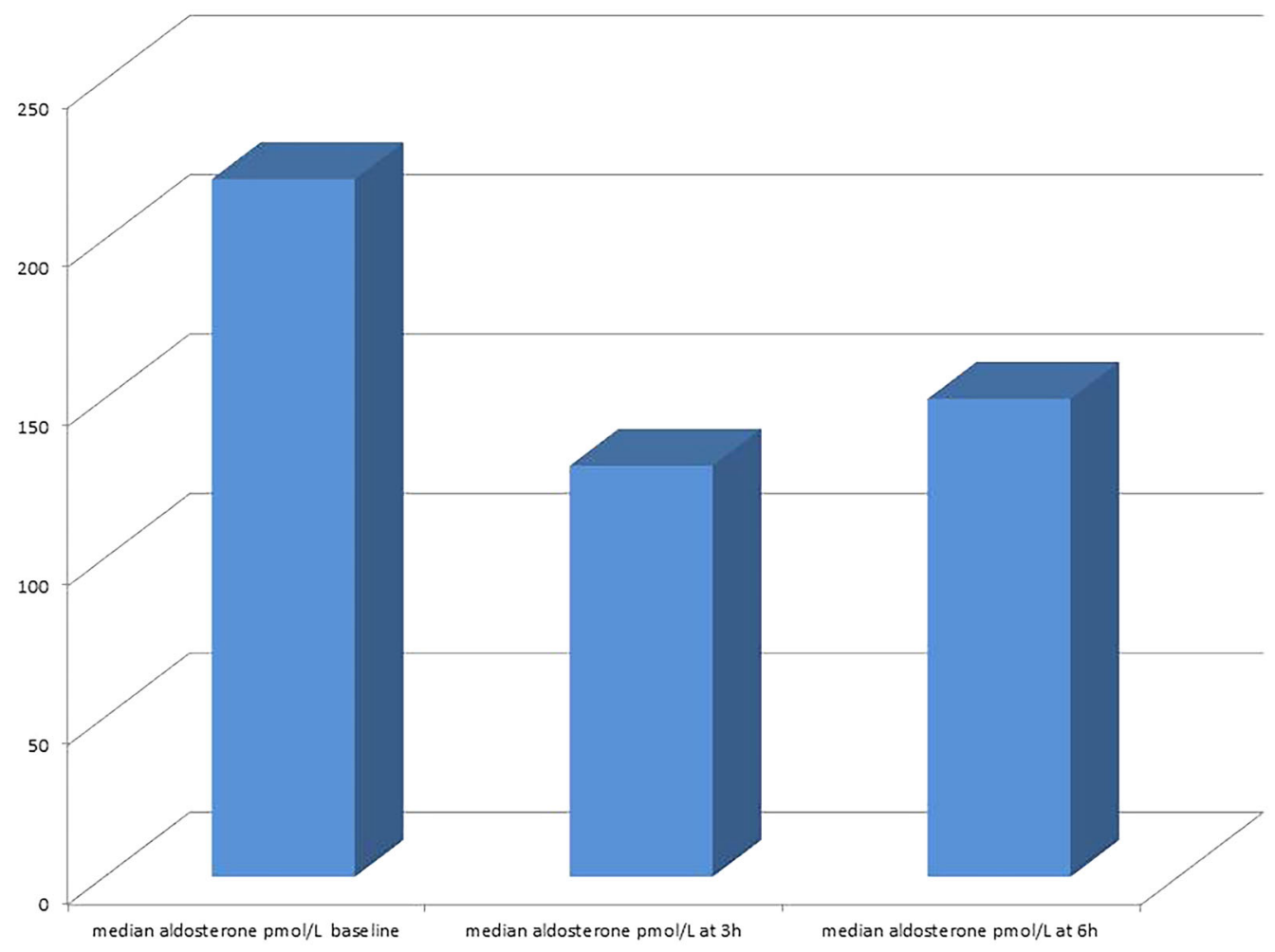

Fig. 2 Suppression of aldosterone following infusion of GLP-1

$3 \mathrm{~h}$ after placebo infusion compared to the baseline insulin level or the insulin level $6 \mathrm{~h}$ after GLP-1 infusion compared to the baseline insulin level) and any of the previously mentioned laboratory and anthropometric variables (age, average systolic blood pressure, average diastolic blood pressure, overnight systolic dipper and overnight diastolic dipper percentages, TSH, glucose in OGTT (glucose at first or second time point), basal value of GLP-1, BMI, mean fat percentage, fat mass, muscle mass, VFR, or TBW).

There was no significant difference between healthy males and females in any observed parameter (GLP-1, aldosterone, PRA, glucose, insulin, or urine sodium) at any follow-up point (all $p>0.05$ ).

\section{DISCUSSION}

We demonstrated that GLP-1 infusion significantly decreased aldosterone after $3 \mathrm{~h}$ in 14 healthy participants, confirming the influence of incretins on the RAAS.

Skov et al. [13] demonstrated that GLP-1 significantly reduced the circulating concentration of angiotensin II by $19 \%$, without producing significant changes in either PRA or the aldosterone level. This effect was attributed to the direct action of GLP-1 on the juxtaglomerular apparatus through changes in renal dynamics and/or the downregulation of angiotensin II production in GLP-1-responsive tissues (i.e., local RAAS cellular production) [15]. One possible explanation for the downregulation of angiotensin II by GLP-1 is the inhibition of angiotensin II through the action of GLP-1 on protein kinase A-dependent phosphorylation. In our study, the decrease in aldosterone $3 \mathrm{~h}$ after GLP-1 infusion may be explained by the direct action of GLP-1 on its receptors in kidney tissue and the inhibition of angiotensin II. In a study by Sedam et al. [16], the long-term administration of liraglutide increased aldosterone levels. That study showed that 
aldosterone tended to increase after chronic dosing with liraglutide, yet the aldosterone-toPRA ratio remained unchanged. Although existing studies have conflicting results, they indicate an association between GLP-1 and the RAAS.

In a large randomized controlled trial comprising 9340 patients with type 2 diabetes and a high cardiovascular risk, liraglutide was compared to placebo (added to standard diabetes care, followed for 3.84 years) [17]. The liraglutide group had lower rates of development and progression of diabetic kidney disease as well as lower rates of new-onset persistent microalbuminuria. The benefits of liraglutide to the kidney are consistent with its known effects on traditional risk factors for progressive kidney disease, including its ability to reduce glucose, blood pressure, insulin levels, and body weight, but it seems likely that something else contributes to these effects as well-perhaps the suppression of the RAAS.

Suppressing the RAAS in type $2 \mathrm{DM}$ patients with hypertension and normoalbuminuria using angiotensin-converting enzyme inhibitors or angiotensin receptor blockers decreased the risk of developing microalbuminuria [18] in a manner similar to that seen in the liraglutide trial. Since new-onset microalbuminuria is an early indicator of diabetic nephropathy, administration of GLP-1 agonists delayed the onset of chronic kidney disease.

GLP-1 infusion caused a decrease in insulin after $3 \mathrm{~h}$. Native GLP-1 in the serum decreases rapidly (with a half-life of several minutes) due to the presence of the enzyme dipeptidyl peptidase IV (DPP-IV) [19]. GLP-1 acts on $\beta$ cells shortly after it has been infused, which is why the effect was observed after $3 \mathrm{~h}$ but not after $6 \mathrm{~h}$. This is also in agreement with the observation that GLP-1 enhances insulin secretion in healthy people [20]. The decreased insulin levels following placebo infusion after $6 \mathrm{~h}$ of fasting (participants fasted overnight and then ingested only salty soup and water during the trial) was an expected result and a consequence of decreased blood glucose due to the prolonged fasting state.

\section{Limitations}

The sample size of the study was relatively small. It was limited due to the expense of the study protocol, the testing procedures applied, and the availability of participants, since repeated measures were needed to obtain data. Hemodynamic and renal function parameters were not evaluated (i.e., the effect on blood pressure, heart rate, changes in albuminuria or renal function). However, GLP-1 infusion into a healthy individual has only a short-term effect, so it is unlikely that these parameters would have changed significantly.

\section{CONCLUSION}

Glucagon-like peptide-1 receptor agonists are widely used as effective and safe antidiabetic medications. As well as their effects on glucosedependent insulin secretion, they play other roles. Their positive influence on blood pressure and the development of kidney disease is attributed to many different mechanisms, not only to glycemic control. There have not been many studies of the basic mechanistic outcome of native GLP-1. The present study showed that native GLP-1 can decrease aldosterone secretion in a group of healthy individuals, supporting the idea that GLP-1-activating agents have beneficial outcomes on blood pressure and the RAAS.

\section{ACKNOWLEDGEMENTS}

We thank the participants of the study.

Funding. This work was supported by the MSD d.o.o., research project grant S15100-NUC, which also covered article processing charges.

Authorship. All named authors meet the International Committee of Medical Journal Editors (ICMJE) criteria for authorship for this article, take responsibility for the integrity of the work as a whole, and have given their approval for this version to be published. 
Disclosures. None of the authors (Maja Baretić, Vesna Kušec, Ivana Pavlić-Renar) have any personal, financial, commercial, or academic conflicts of interest to disclose.

Compliance with Ethics Guidelines. All procedures performed in studies involving human participants were in accordance with the ethics committee of University Hospital Centre Zagreb and with the 1964 Helsinki Declaration and its later amendments or comparable ethical standards. Informed consent was obtained from all individual participants included in the study.

Data Availability. The datasets obtained and/or analyzed during the current study are available from the corresponding author on reasonable request.

Open Access. This article is distributed under the terms of the Creative Commons Attribution-NonCommercial 4.0 International License (http://creativecommons.org/licenses/ by-nc/4.0/), which permits any noncommercial use, distribution, and reproduction in any medium, provided you give appropriate credit to the original author(s) and the source, provide a link to the Creative Commons license, and indicate if changes were made.

\section{REFERENCES}

1. Rabkin R. Diabetic nephropathy. Clin Cornerstone. 2003;5:1-11.

2. Körner M, Stöckli M, Waser B, Reubi JC. GLP-1 receptor expression in human tumors and human normal tissues: potential for in vivo targeting. J Nucl Med. 2007;48:736-43.

3. Badal SS, Danesh FR. New insights into molecular mechanisms of diabetic kidney disease. Am J Kidney Dis. 2014;63:S63-83.

4. Okerson T, Yan P, Stonehouse A, Brodows R. Effects of exenatide on systolic blood pressure in subjects with type 2 diabetes. Am J Hypertens. 2010;23:334-9.

5. Anagnostis P, Athyros VG, Adamidou F, Panagiotou A, Kita M, Karagiannis A, Mikhailidis DP. Glucagon- like peptide-1-based therapies and cardiovascular disease: looking beyond glycaemic control. Diabetes Obes Metab. 2011;13:302-12.

6. Chaudhuri A, Ghanim H, Vora M, Sia CL, Korzeniewski $\mathrm{K}$, Dhindsa $\mathrm{S}$, et al. Exenatide exerts a potent antiinflammatory effect. J Clin Endocrinol Metab. 2012;97:198-207.

7. Fujita H, Morii T, Fujishima H, Sato T, Shimizu T, Hosoba $\mathrm{M}$, et al. The protective roles of GLP-1R signaling in diabetic nephropathy: possible mechanism and therapeutic potential. Kidney Int. 2014;85:579-89.

8. Malendowicz LK, Neri G, Nussdorfer GG, Nowak KW, Zyterska A, Ziolkowska A. Prolonged exendin-4 administration stimulates pituitary-adrenocortical axis of normal and streptozotocin-induced diabetic rats. Int J Mol Med. 2003;12:593-6.

9. Ryan D, Acosta A. GLP-1 receptor agonists: nonglycemic clinical effects in weight loss and beyond. Obesity (Silver Spring). 2015;23:1119-29.

10. Skov J. Effects of GLP-1 in the kidney. Rev Endocr Metab Disord. 2014;15:197-207.

11. Gutzwiller JP, Tschopp S, Bock A, Zehnder CE, Huber AR, Kreyenbuehl M, et al. Glucagon-like peptide 1 induces natriuresis in healthy subjects and in insulin-resistant obese men. J Clin Endocrinol Metab. 2004;89:3055-61.

12. Gutzwiller JP, Hruz P, Huber AR, Hamel C, Zehnder C, Drewe J, et al. Glucagon-like peptide-1 is involved in sodium and water homeostasis in humans. Digestion. 2006;73:142-50.

13. Skov J, Dejgaard A, Frøkiær J, Holst JJ, Jonassen T, Rittig S, et al. Glucagon-like peptide-1 (GLP-1): effect on kidney hemodynamics and renin-angiotensin-aldosterone system in healthy men. J Clin Endocrinol Metab. 2013;98:E664-71.

14. Jelaković B, Kaić-Rak A, Milicić D, Premuzić V, Skupnjak B, Reiner Z. Less salt-more health. Croatian Action on Salt and Health (CRASH). Lijec Vjesn. 2009;131:87-92.

15. Mima A, Hiraoka-Yamomoto J, Li Q, Kitada M, Li C, Geraldes P, et al. Protective effects of GLP-1 on glomerular endothelium and its inhibition by PKCbeta activation in diabetes. Diabetes. 2012;61:2967-79.

16. Sedman T, Heinla K, Vasar E, Volke V. Liraglutide treatment may affect renin and aldosterone release. Horm Metab Res. 2017;49:5-9.

17. Mann JFE, Ørsted DD, Brown-Frandsen K, Marso SP, Poulter NR, Rasmussen S, Tornøe K, Zinman B, Buse 
JB, LEADER Steering Committee and Investigators. Liraglutide and renal outcomes in type 2 diabetes. N Engl J Med. 2017;377:839-48.

18. Persson F, Lindhardt $\mathrm{M}$, Rossing $\mathrm{P}$, Parving $\mathrm{HH}$. Prevention of microalbuminuria using early intervention with renin-angiotensin system inhibitors in patients with type 2 diabetes: a systematic review. J Renin Angiotensin Aldosterone Syst. 2016;3:17.

19. Hansen L, Deacon CF, Orskov C, Holst JJ. Glucagon-like peptide-1-(7-36) amide is transformed to glucagon-like peptide-1-(9-36) amide by dipeptidyl peptidase IV in the capillaries supplying the L cells of the porcine intestine. Endocrinology. 1999;140:5356-63.

20. D'Alessio DA, Kahn SE, Leusner CR, Ensinck JW. Glucagon-like peptide 1 enhances glucose tolerance both by stimulation of insulin release and by increasing insulin-independent glucose disposal. J Clin Investig. 1994;93:2263-6. 